16. As applied to these groups, Theorem 1 can now be stated in the following more significant form :

THEOREM 6 . In an $n$-ary Jordan group, modulo $p^{a}$, for which $p>n+1$, the commutator of a matrix of period $p^{b}$ and a matrix of period $p^{c}$ is a matrix whose period is $p^{b+c-a}$ or a lower power of $p$, if $b+c \geqq a$, while if $b+c \leqq a$, every matrix of period $p^{b}$ is commutative with every matrix of period $p^{c}$.

Under the same restriction that $p>n+1$ we have the

Corollaries: In the subgroup generated by the matrices of period $p^{b}(b \geqq a / 2)$, every matrix of period $p^{a-b}$ is invariant. The commutator of any two matrices of period $p^{b}(b \geqq a / 2)$ is a matrix whose period is $p^{2 b-a}$ or a lower power of $p$; while if $b \leqq a / 2$, any two matrices of period $p^{b}$ are commutative. If $b \leqq a / 2$, the matrices whose periods are divisors of $p^{b}$ form an abelian subgroup of $G$.

Cornele University,

December, 1906.

\title{
ON THE CONSTRUCTION OF AN INTEGRAL OF LAGRANGE'S EQUATIONS IN THE CALCULUS OF VARIATIONS.
}

BY DR. D. C. GILLESPIE.

( Read before the American Mathematical Society, December 29, 1906.)

1. Darboux * has proven the theorem :

When (1) $\int_{1}^{2} F\left(y^{\prime}, y, x\right) d x \quad$ and (2) $\int_{1}^{2} F^{\prime}\left(y^{\prime}, y, x\right) d x$

lead to the same Lagrange equation; $i . e$. , when the first variation of integrals (1) and (2) equated to zero give the same differential equation (3) $y^{\prime \prime}=\omega\left(y^{\prime}, y, x\right)$, where $y^{\prime \prime}=d^{2} y / d x^{2}$, then $F_{y^{\prime} y^{\prime}} / F^{\prime \prime} y^{\prime} y^{\prime}=$ const. (literal subscripts denote partial derivatives) is an intermediary of equation (3).

2. This theorem can be proven with very little algebraic work by making use of Hilbert's $\dagger$ independence theorem (Unabhängigkeitssatz).

* Darboux, Théorie des surfaces, vol. 3, pp. 59-65.

† Hilbert's Vorlesungen über Variationsrechnung, Winter-Semester, 19041905 . 
This latter method lends itself readily to the investigation of the problem for a system of differential equations.

$$
\text { Let (5) } \int_{1}^{2} F\left(z^{\prime}, z, y^{\prime}, y, x\right) d x \text { and (6) } \int_{1}^{2} F^{\prime}\left(z^{\prime}, z, y^{\prime}, y, x\right) d x \text {, }
$$

be two integrals, the vanishing of the first variation of which leads to the same system of differential equations

$$
y^{\prime \prime}=\omega_{1}\left(z^{\prime}, z, y^{\prime}, y, x\right), \quad z^{\prime \prime}=\omega_{2}\left(z^{\prime}, z, y^{\prime}, y, x\right) .
$$

The purpose here is to show that

$$
\left|\begin{array}{cc}
F_{y^{\prime} y^{\prime}}, & F_{y^{\prime} z^{\prime}} \\
F_{y^{\prime} z^{\prime}}, & F_{z^{\prime} z^{\prime}}
\end{array}\right| \div\left|\begin{array}{ll}
F_{y^{\prime} y^{\prime}}^{\prime \prime} & F_{y^{\prime} z^{\prime}}^{\prime} \\
F_{y^{\prime} z^{\prime}}^{\prime} & F_{z^{\prime} z^{\prime}}^{\prime}
\end{array}\right|=\text { const. }
$$

is an integral of the system (4) (in case of course the quotient itself is not identically constant).

The method of proof for a system of two differential equations gives at once the result for a system of $m$ equations.

Out of the integrals (5) and (6) are built the integrals

$$
\begin{gathered}
\int_{1}^{2}\left\{F(q, z, p, y, x)+\left(y^{\prime}-p\right) F_{p}+\left(z^{\prime}-q\right) F_{q}\right\} d x \\
\int_{1}^{2}\left\{F^{\prime}(q, z, p, y, x)+\left(y^{\prime}-p\right) F_{p}^{\prime}+\left(z^{\prime}-q\right) F_{q}^{\prime}\right\} d x .
\end{gathered}
$$

The method requires that there exist a $p(x, y, z, \alpha, \beta), q(x, y, z$, $\alpha, \beta)$ (where $\alpha$ and $\beta$ are arbitrary constants) which make integrals $\left(5^{\prime}\right)$ and $\left(6^{\prime}\right)$ independent of the path of integration. Although not every pair of functions $p(x, y, z), q(x, y, z)$ which render $\left(5^{\prime}\right)$ independent of the path will necessarily render $\left(6^{\prime}\right)$ independent of the path,* yet it follows from the results obtained by Mayer $\uparrow$ that there exists a two-parameter system of functions $p(x, y, z, q, \alpha, \beta), q(x, y, z, \alpha, \beta)$ which makes both integrals independent of the path of integration. We may assume therefore two such functions, $p$ and $q$; it follows then that the integrand of each of the integrals $\left(5^{\prime}\right)$ and $\left(6^{\prime}\right)$ is an exact differential for all values of $\alpha$ and $\beta$ and hence their derivatives with respect to $\alpha$ and $\beta$ will be exact differen-

* There is an example showing this in my thesis, "Anwendungen des Unabhängigkeitssatzes auf die Lösung der Differentialgleichungen der Variationsrechnung" (Göttingen 1906).

† Math. Annalen, vol. 58. 
tials. Differentiating $\left(5^{\prime}\right)$ with respect to $\alpha$ and $\beta$ and equating to zero, we obtain the two exact differential equations

(7) $\left(y^{\prime}-p\right)\left(F_{p p} p_{a}+F_{p q} q_{a}\right)+\left(z^{\prime}-q\right)\left(F_{p q} p_{a}+F_{q q} q_{a}\right)=0$,

(8) $\left(y^{\prime}-p\right)\left(F_{p p} p_{\beta}+F_{p q} q_{\beta}\right)+\left(z^{\prime}-q\right)\left(F_{p q} p_{\beta}+F_{q q} q_{\beta}\right)=0$.

If $B$ and $C$ represent the coefficients of $y^{\prime}$ and $z^{\prime}$ in equation (7), then the remaining terms $A$ are $-p B-q C$; in the same way, $B_{1}, C_{1}$ and $A_{1}$ representing corresponding members of equation (8), $A_{1}=-p B_{1}-q C_{1}$. The equations being exact, $A_{y}=B_{x}, A_{z}=C_{x}, B_{z}=C_{y}$ and $A_{1 y}=B_{1 x}, A_{1 z}=C_{1 x}, B_{1 z}=C_{1 y}$.

The identity

$$
\frac{\partial}{\partial x}\left|\begin{array}{cc}
B & B_{1} \\
C & C_{1}
\end{array}\right|+\frac{\partial}{\partial y} p\left|\begin{array}{cc}
B & B_{1} \\
C & C_{1}
\end{array}\right|+\frac{\partial}{\partial z} q\left|\begin{array}{ll}
B^{\prime} & B_{1}^{\prime} \\
C^{\prime} & C_{1}^{\prime}
\end{array}\right| \equiv 0
$$

therefore follows. This method applied to integral $\left(6^{\prime}\right)$ gives the second identity

$$
\frac{\partial}{\partial x}\left|\begin{array}{ll}
B^{\prime} & B_{1}^{\prime} \\
C^{\prime} & C_{1}^{\prime}
\end{array}\right|+\frac{\partial}{\partial y} p\left|\begin{array}{ll}
B^{\prime} & B_{1}^{\prime} \\
C^{\prime} & C_{1}^{\prime}
\end{array}\right|+\frac{\partial}{\partial z} q\left|\begin{array}{ll}
B & B_{1} \\
C & C_{1}
\end{array}\right| \equiv 0,
$$

where $B^{\prime}, B_{1}^{\prime}, C_{1}^{\prime}, C_{1}^{\prime}$ have corresponding meanings.

If we denote by $m(x, y, z, p, q)$ the quotient

$$
\left|\begin{array}{ll}
B & B_{1} \\
C & C_{1}
\end{array}\right| \div\left|\begin{array}{ll}
B^{\prime} & B_{1}^{\prime} \\
C^{\prime} & C_{1}^{\prime}
\end{array}\right|
$$

we obtain for $m$ the identity in $x, y, z, \alpha$ and $\beta$

$$
\begin{gathered}
\frac{\partial m}{\partial x}+p \frac{\partial m}{\partial y}+q \frac{\partial m}{\partial z} \equiv 0 \\
{[p=p(x, y, z, \alpha, \beta), \quad q=q(x, y, z, \alpha, \beta)] .} \\
m \equiv\left|\begin{array}{ll}
F_{p p}^{\prime} & F_{p q}^{\prime} \\
F_{p q} & F_{q q}^{\prime}
\end{array}\right| \div\left|\begin{array}{ll}
F_{p_{p}}^{\prime} & F_{p_{q}}^{\prime \prime} \\
F_{p q}^{\prime} & F_{q q}^{\prime}
\end{array}\right|
\end{gathered}
$$

is a known function of $x, y, z, p$ and $q$.

$$
y^{\prime}=p(x, y, z, \alpha, \beta), \quad z^{\prime}=q(x, y, z, \alpha, \beta)
$$


is moreover according to the independence theorem an intermediary integral of the system of equations (4). If now we choose any particular solution of equations (4) $y=\bar{y}(x), z=\bar{z}(x)$, then a pair of values of $\alpha$ and $\beta$ always exists, which we will write $\alpha_{0}, \beta_{0}$, such that $p\left(x, \bar{y}(x), \bar{z}(x), \alpha_{0}, \beta_{0}\right) \equiv \bar{y}(x), q(x, \bar{y}(x)$, $\left.\bar{z}(x), \alpha_{0}, \beta_{0}\right) \equiv \bar{z}(x)$. When we substitute for $y, z, p$ and $q$ in the identity (11) the functions $\bar{y}(x), \bar{z}(x), \bar{y}^{\prime}(x), \bar{z}^{\prime}(x)$, we obtain an identity in $x$; but (11) becomes in this case the total differential quotient of $m$ with respect to $x$.

$$
\therefore \frac{d m}{d x}=0, m=\text { const. }
$$

Since $\bar{y}(x), \bar{z}(x)$ were chosen arbitrarily, every solution of the system of equations (4) substituted in $m$ gives a constant; hence

$$
m\left(x, y, z, y^{\prime}, z^{\prime}\right) \equiv\left|\begin{array}{ll}
F_{y^{\prime} y^{\prime}} & F_{y^{\prime} z^{\prime}} \\
F_{z^{\prime} y^{\prime}} & F_{z^{\prime} z^{\prime}}
\end{array}\right| \div\left|\begin{array}{cc}
F_{y^{\prime} y^{\prime}}^{\prime} & F_{y^{\prime} z^{\prime}}^{\prime} \\
F_{y^{\prime} z^{\prime}}^{\prime} & F_{z^{\prime} z^{\prime}}^{\prime}
\end{array}\right|=\text { const. }
$$

is an integral of the system (4).

A substitution which leaves system (4) invariant transforms the integral of which (4) are the Lagrange equations either into itself or into a new integral which has the same Lagrange equations. In this latter case, the one integral being given, the construction of such a substitution is equivalent to the construction of an integral.

CoRneld UNIVERSITY, December, 1906 .

\section{ALGEBRAIC NUMBERS AND FORMS.}

Zahlentheorie. Fünfter Teil : Allgemeine Arithmetik der Zahlenkörper. By Paul Bachmann. Leipzig, B. G. Teubner, 1905. xxii +548 pp.

Einleitung in die allgemeine Theorie der Algebraischen Grössen.

By Julius Könıg. Leipzig, B. G. Teubner, 1903. $\mathrm{x}+564$

pp.

There has been but little activity in America in this important and fascinating field. It seems appropriate, therefore, to preface this review with an elementary introduction to the subject. We shall consider the simpler features of the theory of quadratic number systems, for which the phenomena are 